\title{
OBESIDAD Y ANEMIA EN MUJERES EMBARAZADAS A BAJA Y GRAN ALTITUD
}

\author{
Paola Olavegoya $^{1}$, Gustavo F. Gonzales ${ }^{1}$
}

\begin{abstract}
RESUMEN
Objetivo. Demostrar si la obesidad pregestacional en mujeres embarazadas de poblaciones que viven a bajas y altas altitudes se relaciona con la reducción de la hemoglobina $(\mathrm{Hb})$ y si se observan cambios en cada uno de los trimestres del embaraø. Métodos. Utilizamos una base de datos que contiene información de 1'712,639 mujeres embarazadas pertenecientes a cada uno de los 24 departamentos del Perú obtenidas durante el período 2012 a 2017 . Se determinó el peso y la talla antes del embarazo, y se calculó el IMC y se expresó como el peso en kilogramos dividido por el cuadrado de altura en metros. También se registraron los datos sobre el aumento de peso durante el embarazo. Las mediciones de hemoglobina se obtuvieron mediante el dispositivo hemocue. Los datos se presentan como la concentración de Hb no corregida $(\mathrm{g} / \mathrm{dL})$ y la concentración de $\mathrm{Hb}$ corregida $(\mathrm{g} / \mathrm{dL})$ después de ajustar su valor por altitud según lo recomendado por la Organización Mundial de la Salud (OMS). Los datos se analizaron mediante análisis bivariados y multivariados. P $<0,05$ fue considerado como significativo. Resultados. Conforme aumentó la categoría de IMC aumentó la concentración de hemoglobina (corregida y no corregida) $(p<0,0001)$ mientras que la ganancia de peso durante el embarazo se reducía en forma de dosis-respuesta a medida que aumentaba el IMC $(P<0,0001)$. Se encontraron diferencias significativas en la edad (años) y el IMC $(\mathrm{Kg} / \mathrm{m} 2)$ entre todos los grupos con respecto al trimestre $(p<0,0001)$. Las mujeres con sobrepeso y obesidad no aumentan de peso en el primer trimestre del embarazo. La obesidad se asoció con un bajo aumento de peso en el segundo y tercer trimestre del embarazo. El mayor aumento de peso durante el embarazo se correlacionó con una menor concentración de hemoglobina $\left(R^{2}=0,963 ; p<0,0001\right)$. Conclusiones. Es necesario medir el volumen plasmático de las gestantes con la finalidad de evitar malinterpretaciones de la real concentración de hemoglobina en gestantes con obesidad y sobrepeso.
\end{abstract}

Palabras clave: Obesidad; Anemia; Embarazo; Hemoglobina (Fuente: DeCS BIREME).

\section{OBESITY AND ANEMIA IN PREGNANT WOMEN AT LOW AND HIGH ALTITUDE}

\begin{abstract}
Objective. To demonstrate if pregestational obesity in populations living at low and at high altitudes was associated with hemoglobin $(\mathrm{Hb})$ reduction and if changes are observed in each of the trimesters of pregnancy. Methods. We used a database containing information from 1'712,639 pregnant women belonging to each of the 24 departments of Peru obtained during the period 2012 to 2017. Pre-pregnancy Weight and height were determined, and BMI was calculated and expressed as weight in kilograms divided by the square of height in meters. Data about weight gain during pregnancy was also recorded. Hemoglobin was measured using hemocue. Data are presented as uncorrected hemoglobin concentration $(\mathrm{g} / \mathrm{dL})$ as well as corrected hemoglobin concentration ( $\mathrm{g} / \mathrm{dL}$ ) after adjusting its value by altitude as recommended by World Health Organization. Data were analyzed using bivariate and multivariate analysis. $\mathrm{P}<0,05$ was considered as significant. Results. Increase of BMI category increased the hemoglobin concentration (corrected or uncorrected) $(p<0,0001)$ whereas, the weight gain from pregnancy is lowered in a dose-response fashion as BMI category increased $(P<0,0001)$. Significant differences were found in age (years) and BMI $\left(\mathrm{Kg} / \mathrm{m}^{2}\right)$ between all groups regarding trimester $(p<0,0001)$. Women with overweight and obesity does not increase weight at first trimester of pregnancy. Obesity was associated with low weight gain at second and third trimester of pregnancy. Higher pregnancy weight gain was correlated with lower hemoglobin concentration $\left(R^{2}=0,963 ; p<0,0001\right)$. Conclusions. It is necessary to measure the plasma volume of pregnant women, as this would avoid misinterpretations of hemoglobin concentration in overweight and obese pregnant women.
\end{abstract}

Keywords: Obesity; Anemia; Pregnancy, hemoglobin (Source: MeSH NLM).

\section{INTRODUCCIÓN}

La obesidad es considerada un proceso inflamatorio. Varios estudios han demostrado que la obesidad y la inflamación aumentan los niveles séricos de hepcidina, una hormona producida en el hígado que regula la disponibilidad de hierro en el cuerpo.
Un aumento en el nivel de hepcidina en suero después de la inflamación se asocia con una baja absorción de hierro intestinal y baja liberación de macrófagos, esto ha sido asoc ado o $\mathrm{n}$ un maø $\mathrm{r}$ riesgo de desarrollar anemia (Del Giudie et al 2009). La relac ón entre la obesidad y el riesgo de anemia ha sido observado claramente en niños (Nead et al 2004). Sin embargo, algunos estudios en

\footnotetext{
Laboratorio de Endocrinología y Reproducción, Laboratorios de Investigación y Desarrollo (LID), Facultad de Ciencias y Filosofía, e Instituto de Investigaciones de la Altura. Universidad Peruana Cayetano Heredia

Citar como: Olavegoya P, Gonzales GF. Obesidad y anemia en mujeres embarazadas a baja y gran altitud. Rev Peru Investig Matern Perinat $2018 ; 7(1): 18-23$. DOI https://doi.org/10.33421/inmp.2018105
}

Recibido: 10-06-18 Aprobado: 30-06-18 
mujeres embarazadas han observado que la obesidad se asoć aba más bien o $\mathrm{n}$ un aumento en la o ne ntrac ón de hemoglobina en lugar de una reducción (Vricella et al 2015).

A diferencia de lo que sucede en niños, en mujeres embarazadas la concentración de $\mathrm{Hb}$ depende de la cantidad del volumen de expansión. La disminución de los valores de hemoglobina durante el segundo y tere $r$ trimestre del embarazo se debe a un fenómeno llamado hemodilución (West et al 2016). Este proceso puede variar y producir enfermedades durante el embarazo. Por ejemplo, la preeclampsia está asociada con la hemoo ne ntrac ón y o $\mathrm{n}$ un aumento en la o ne ntrac ón de la hemoglobina, siendo un factor de riesgo para la madre y el feto (Khoigani y col, 2012).

La hemodilución durante el embarazo es un proceso de suma importancia pues favorece el flujo uterino-placentario aumentando la disponibilidad de oxígeno y nutrientes para el feto (Dimasuay et al 2016). En poblaciones a gran altura se ha observado una reducción en el volumen plasmático lo cual puede llevar a producir hemoconcentración. Las mujeres embarazadas con altos niveles de hemoglobina tienen mayor riesgo de tener partos con niños pequeños y prematuros para la edad gestacional (Gonzales et al 2014).

Tenemos una base de datos importante de 1'712,639 mujeres embarazadas a las cuales se le midió la hemoglobina en poblaciones de diferentes altitudes en Perú durante el período 2012 al 2017. Con este tamaño de muestra, hemos decidido demostrar si el sobrepeso/ obesidad en mujeres embarazadas está asociado con una reducción de la $\mathrm{Hb}$ y aumentando el riesgo de anemia y si se observan a mbios en a da uno de los trimestres del embaraø.

\section{MATERIALES Y MÉTODOS}

Este fue un análisis secundario de una base de datos recopilada durante el período 2012-2017 que cumple con una norma emanada por el Ministerio de Salud (MINSA) de Perú. Los datos se recolectaron en diferentes distritos y provincias de cada uno de los 24 departamentos del Perú a través de la Direć ón Regional de Salud. Estos datos se enviaron al Centro Nacional de Alimentación y Nutrición (CENAN) ubicado en Lima, la capital de Perú. CENAN es un organismo perteneciente al Instituto Nacional de Salud en el Perú. La base de datos fue enviada por el Jefe del INS, Perú a la Universidad Peruana Cayetano Heredia para investigación.

Se revisó la base de datos y se eliminaron los datos discordantes. El Comité de Ética de la Universidad Peruana Cayetano Heredia aprobó el estudio. La base de datos incluyó las siguientes variables: edad, edad gestacional, peso pre-gestacional, peso gestacional, ganancia de peso durante el embarazo, IMC pre-gestacional, altitud, hemoglobina corregida y no corregida en la fecha de la toma de la muestra que puede ser el primer, segundo o tere $r$ trimestre de gestaic ón.

Se determinó el peso antes del embarazo, el peso y la altura de la embarazada. Se calculó el IMC y se expresó como el peso en kilogramos dividido por el cuadrado de la altura en metros. El IMC se clasificó en seis grupos: bajo peso (<18.5 kg/m2), peso normal (18.5-24.9 kg/m2), sobrepeso (25-29.9 kg/m2), obesidad clase I (30-34.99 $\mathrm{kg} / \mathrm{m} 2)$, obesidad clase II $(35-39.99 \mathrm{Kg} / \mathrm{m} 2)$ y obesidad clase III ( $\geq 40 \mathrm{Kg} / \mathrm{m} 2)$ según lo recomendado por la OMS para obesidad durante el embarazo (OMS, 2004). Las medic ones de hemoglobina se obtuvieron mediante un equipo de hemocue. Los datos se presentaron como concentración de hemoglobina no corregida $(\mathrm{g} / \mathrm{dL})$ y como hemoglobina corregida después de ajustar su valor por altitud según lo recomendado por la OMS (Gonzales et al 2014; OMS, 2001).

La base de datos inicial fue de 1'787,214 datos, eliminamos los datos aberrantes y utilia mos un total de 1'712,639 datos de mujeres embarazadas. La edad media fue de 25.72 años (Min 12, Max 49), el IMC promedio fue de $24.73 \mathrm{~kg} / \mathrm{m} 2$ (Min. 9.94, Max 62.93), la media de la concentración de hemoglobina no corregida fue de 12.44 $\mathrm{g} / \mathrm{dL}$ (Min 4, Max 21.8) y la ganancia de peso promedio en el momento de la evaluación fue de $5.45 \mathrm{~kg}$ (Min -10.8, Max 20). De la muestra estudiada, el $9.59 \%$ correspondió a mujeres embarazadas en el primer trimestre, $32.34 \%$ en el segundo trimestre y $58.05 \%$ en el tercer trimestre.

El porcentaje de anemia leve en mujeres embarazadas fue del $13.06 \%$, la anemia moderada fue del $2.99 \%$, la anemia severa fue del $0.04 \%$ para hemoglobina no corregida y el $24.85 \%$ anemia leve, $8.02 \%$ anemia moderada y $0.18 \%$ anemia severa para los valores de hemoglobina corregida. Probamos las diferencias en las características maternas categóricas según IMC utilizando la prueba $\mathrm{X}^{2}$, así mismo, utilizamos la prueba de ANOVA de una vía para evaluar las diferencias en los valores medios de las características maternas continuas según el IMC y el trimestre. Utilizamos el análisis multivariado para probar la interacción del aumento de peso con otras variables. Todos los análisis estadísticos se realizaron con STATA versión 12.0.

\section{RESULTADOS}

Del total de mujeres embarazadas evaluadas, $2.13 \%$ $(36,649 / 1 ' 712,639)$ tenían bajo peso previo al embarazo, $57.31 \%(981.606 / 1 ' 712,639)$ tenían peso normal, $30.81 \%(527,708 / 1 ' 712,639)$ tenían sobrepeso y $9.73 \%$ $(166,676 / 1712,639)$ tenía obesidad.

Se evaluaron los niveles de hemoglobina o rregida y no corregida en mujeres embarazadas durante el período de 2012 a 2017 en el Perú clasificados según el estado 
Tabla 1. Niveles de hemoglobina corregida y niveles de hemoglobina no corregida en mujeres embarazadas durante el período de 2012 a 2017 en Perú clasificados según el estado de índice de masa corporal (IMC) preconcepcional.

\begin{tabular}{|c|c|c|c|c|c|c|}
\hline $\begin{array}{c}\text { IMC } \\
\left(\mathrm{Kg} / \mathrm{m}^{2}\right)\end{array}$ & $\begin{array}{l}\text { Edad } \\
\text { (Años) }\end{array}$ & $\begin{array}{c}\text { Edad } \\
\text { Gestac onal } \\
\text { (Semanas) }\end{array}$ & $\begin{array}{c}\text { Altitud } \\
\text { (m.s.n.m) }\end{array}$ & $\begin{array}{l}\text { Hemoglobina no } \\
\text { corregida (g/dL) }\end{array}$ & $\begin{array}{l}\text { Hemoglobina } \\
\text { corregida }(\mathrm{g} / \mathrm{dL})\end{array}$ & $\begin{array}{c}\text { Gananic a de } \\
\text { peso } \\
(\mathrm{Kg})\end{array}$ \\
\hline $\begin{array}{l}\text { Bajo peso } \\
(n=36,649)\end{array}$ & $21.58 \pm 0.06^{*}$ & $27.04 \pm 0.08^{*}$ & $969.92 \pm 6.58^{* d}$ & $12.02 \pm 0.01^{\star d}$ & $11.52 \pm 0.01^{* d}$ & $8.09 \pm 0.04^{\star d}$ \\
\hline $\begin{array}{c}\text { Normal } \\
(n=981,606)\end{array}$ & $24.24 \pm 0.01^{*}$ & $27.17 \pm 0.01^{*}$ & $1399.94 \pm 1.46^{*_{c}}$ & $12.40 \pm 0.01^{* c}$ & $11.57 \pm 0.01^{* c}$ & $6.22 \pm 0.01^{* c}$ \\
\hline $\begin{array}{l}\text { Sobrepeso } \\
(n=527,708)\end{array}$ & $27.55 \pm 0.01^{*}$ & $27.03 \pm 0.01^{*}$ & $1261.77 \pm 1.93^{\star b}$ & $12.52 \pm 0.01^{* b}$ & $11.80 \pm 0.01^{* \mathrm{~b}}$ & $4.68 \pm 0.01^{\star b}$ \\
\hline $\begin{array}{c}\text { Obesidad } \\
\text { Clase I } \\
(n=137574)\end{array}$ & $29.44 \pm 0.01^{*}$ & $26.85 \pm 0.01^{\star a}$ & $982.33 \pm 3.46^{\star a}$ & $12.52 \pm 0.01^{* \mathrm{e}}$ & $12.00 \pm 0.01^{* a}$ & $3.31 \pm 0.01^{* a}$ \\
\hline $\begin{array}{c}\text { Obesidad Clase } \\
\text { II } \\
\qquad(n=24869)\end{array}$ & $29.89 \pm 0.01^{*}$ & $26.70 \pm 0.01^{*}$ & $760.27 \pm 7.26^{* f}$ & $12.46 \pm 0.01^{*}$ & $12.09 \pm 0.01^{* f}$ & $2.37 \pm 0.01^{\star f}$ \\
\hline $\begin{array}{c}\text { Obesidad clase } \\
\text { III } \\
(n=4233)\end{array}$ & $30.13 \pm 0.01^{*}$ & $26.21 \pm 0.01^{*}$ & $559.36 \pm 14.92^{* g}$ & $12.42 \pm 0.01^{*}$ & $12.17 \pm 0.01^{* g}$ & $1.67 \pm 0.01^{* g}$ \\
\hline
\end{tabular}

del índice de masa corporal (Tabla 1). Se encontraron diferencias significativas en la edad (años), la edad gestacional (semanas), la altitud de residencia (m.s.n.m) entre todos los grupos con respecto al IMC $(p<0,0001)$. Contrariamente a la hipótesis, se observó que mientras se inc ementaba el IMC también aumentaba la o ne ntrac ón de hemoglobina (corregida o no) $(p<0,0001)$, mientras que la ganancia de peso durante el embarazo se reducía en forma de dosis-respuesta a medida que aumenta el IMC $(p<0,0001)$.

El análisis multivariado determinó que el aumento de peso del embarazo no dependía del IMC (Coef: 0.34; IC del 95\%: 0.34-0.33) (Tabla 2).

Los datos expresados por la media \pm EE. ANOVA: ${ }^{*} p<0,0001$ entre todos los grupos. a $P<0.0001$ en comparación con los grupos de bajo peso, normal y con sobrepeso. b $\mathrm{P}<0.0001$ en comparación con grupos de bajo peso, normal y obesidad I, II, III. c p $<0.0001$ en comparación con los grupos de bajo peso, sobrepeso y obesidad I, II, III. d p <0,0001 en comparación con los grupos normal, sobrepeso y obesidad I, II, II. e $p<0.04$ en comparación con el grupo con sobrepeso. F p $<0.0001$ comparado con el normal, bajo peso, sobrepeso, obesidad clase I y obesidad clase III. g p $<0.0001$ comparado con bajo peso, normal, con sobrepeso, obesidad clase I y obesidad $\mathrm{k}$ ase II.

Se evaluaron los niveles de hemoglobina o rregida y no corregida en mujeres clasificadas según trimestre de embarazo (tabla 3). Se encontraron diferencias significativas en la edad (años) y IMC $\left(\mathrm{Kg} / \mathrm{m}^{2}\right)$ entre todos los grupos con respecto al trimestre $(p<0,0001)$. La ganancia de peso durante el embarazo fue mayor en el tercer trimestre con respecto a los valores del primer y segundo trimestre (Tabla 3). Dividimos el tercer trimestre de según la ganancia de peso (datos no presentados), el IMC aumentó significativamente en el tercer trimestre del embaraø .

La edad y la altitud fueron significativamente más altas en el primer trimestre del embarazo. Hubo una disminución de la $\mathrm{Hb}$ corregida y no corregida independientemente del IMC, la hemoglobina (corregida y no corregida) se redujo en el segundo trimestre del embarazo y más en el tercer trimestre. La Hb fue más alta en el sobrepeso y la obesidad en a da trimestre del embaraø.

Tabla 2. Análisis multivariado del aumento de peso y el índice de masa corporal (IMC) en mujeres embarazadas durante el período de 2012 a 2017 en Perú.

\begin{tabular}{ccccccc}
\hline & \multicolumn{5}{c}{ Ganancia de peso } \\
\cline { 2 - 7 } Factor & Coef. & Std. Err. & $\mathbf{T}$ & $\begin{array}{c}\text { Valor } \mathbf{p} \\
\mathbf{p}>|\mathbf{t}|\end{array}$ & IC 95 \% \\
\hline Bajo peso & 1.91 & 0.02 & 91.68 & 0.000 & 1.87 & 1.95 \\
Normal & -1.48 & 0.01 & -221.22 & 0.000 & -1.49 & -1.47 \\
Sobrepeso & -2.79 & 0.01 & -247.24 & 0.000 & -2.82 & -2.77 \\
\hline Obesidad clase I & -3.68 & 0.02 & -145.80 & 0.000 & -3.73 & -3.63 \\
Obesidad clase II & -4.22 & 0.06 & -69.74 & 0.000 & -4.34 & -4.10 \\
Obesidad clase III & 0.34 & 0.001 & 1013.57 & 0.000 & 0.34 & 0.34 \\
o ns & -3.03 & 0.001 & -305.01 & 0.000 & -3.05 & -3.01 \\
\hline
\end{tabular}


Tabla 3. Niveles de hemoglobina corregida y no corregida por altitud en mujeres embarazadas durante el período de 2012 a 2017 en Perú por trimestre.

\begin{tabular}{|c|c|c|c|c|c|c|}
\hline TRIMESTRE & $\begin{array}{c}\mathrm{IMC} \\
\left(\mathrm{Kg} / \mathrm{m}^{2}\right)\end{array}$ & $\begin{array}{l}\text { Edad } \\
\text { (Años) }\end{array}$ & $\begin{array}{c}\text { Altitud } \\
\text { (m.s.n.m) }\end{array}$ & $\begin{array}{l}\text { Hemoglobina no } \\
\text { corregida }(\mathrm{g} / \mathrm{dL})\end{array}$ & $\begin{array}{l}\text { Hemoglobina } \\
\text { corregida (g/dL) }\end{array}$ & $\begin{array}{c}\text { Gananic a de } \\
\text { peso } \\
(\mathrm{Kg})\end{array}$ \\
\hline $\begin{array}{c}1^{\text {er }} \\
(n=164396)\end{array}$ & $24.95 \pm 0.01^{a}$ & $26.27 \pm 0.01^{a}$ & $1420 \pm 3.49^{a}$ & $13.03 \pm 0.01^{\mathrm{a}}$ & $12.22 \pm 0.01^{\mathrm{a}}$ & $0.38 \pm 0.01^{a}$ \\
\hline $\begin{array}{c}2^{\text {do }} \\
(n=553978)\end{array}$ & $24.70 \pm 0.01^{b}$ & $25.63 \pm 0.01$ & $1292 \pm 1.91$ & $12.53 \pm 0.01^{b}$ & $11.78 \pm 0.01^{b}$ & $2.83 \pm 0.01^{b}$ \\
\hline $\begin{array}{c}3^{e r} \\
(n=994265)\end{array}$ & $24.71 \pm 0.01^{c}$ & $25.68 \pm 0.01$ & $1290 \pm 1.42$ & $12.29 \pm 0.01^{c}$ & $11.54 \pm 0.01^{c}$ & $7.81 \pm 0.01^{c}$ \\
\hline
\end{tabular}

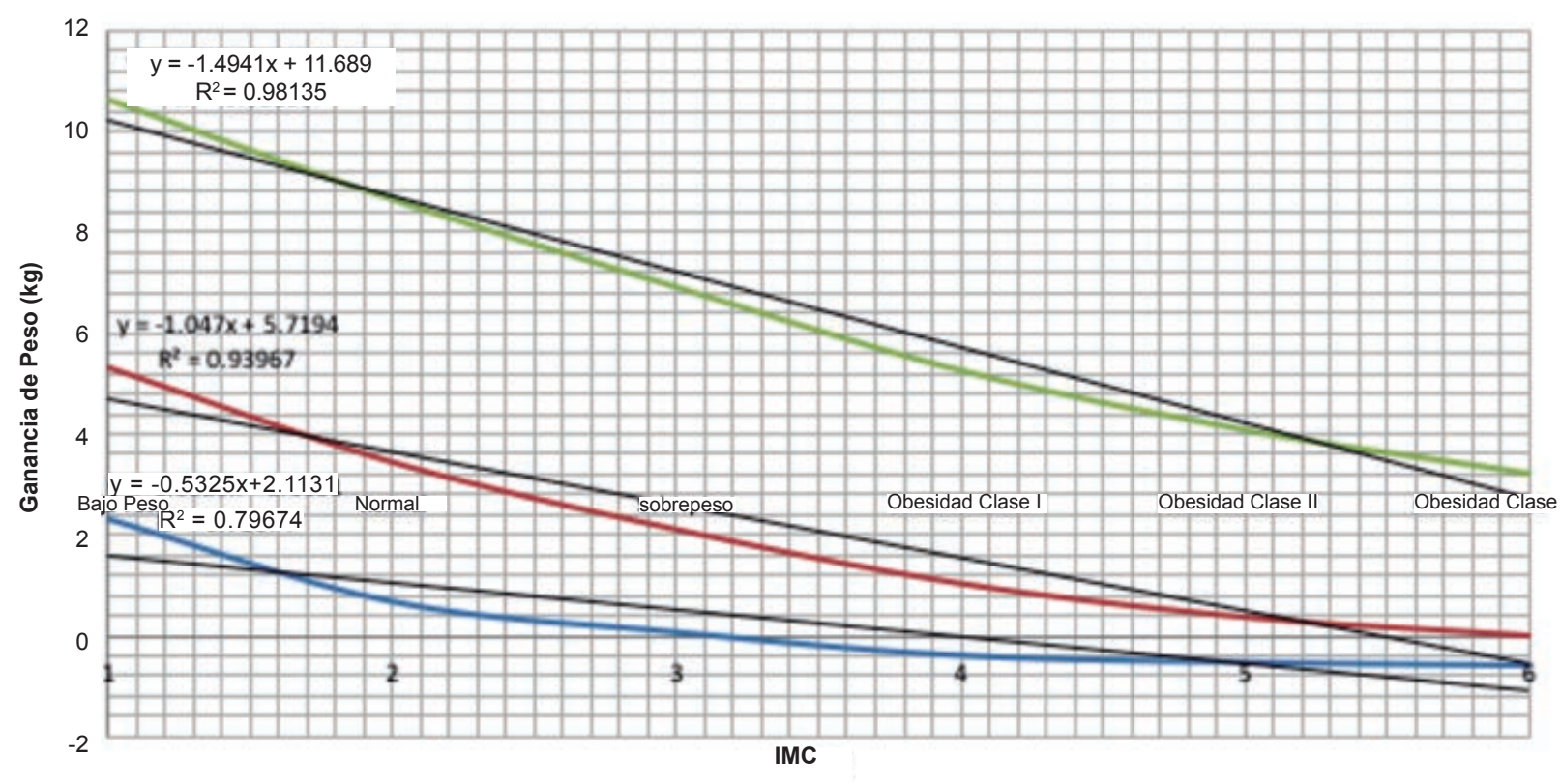

1er trimestre $\quad$ 2do trimestre $\quad-3 e r$ trimestre

Figura 1. Correlación entre la ganancia de peso durante el embarazo y categoría del IMC por trimestre en mujeres embarazadas durante el período de 2012 a 2017 en Perú. I: bajo peso (<18.5 kg/m2), II:peso normal (18.5-24.9 kg/m2), III: sobrepeso (25-29.9 kg/m2), IV: obesidad clase I (30-34.99 kg/m2), V: obesidad clase II (35-39.99 Kg/m2) y VI: obesidad clase III ( $\geq 40 \mathrm{Kg} / \mathrm{m} 2)$.

Los datos fueron expresados por la media \pm EE. ANOVA: $p<0,0001$ entre todos los grupos. a $\mathrm{P}<0.0001$ en comparación con el $2^{\text {do }}$ y $3^{\text {er }}$ trimestre. b $\mathrm{P}<0,0001$ comparado con el $1^{\text {er }}$ y $3^{\text {er }}$ trimestre. c $p<0.0001$ en comparación con el $1^{\text {er }}$ y $2^{\text {do }}$ trimestre.

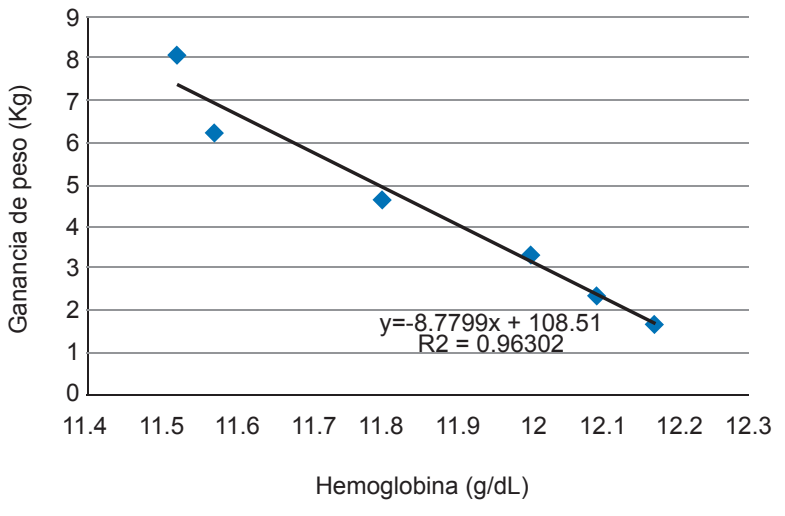

Figura 2. Correlación entre el aumento de peso durante el embarazo y la concentración de hemoglobina. $\mathrm{P}<0.001$
Se observó una mayor ganancia de peso durante el embarazo en mujeres con un IMC bajo (Figura 1). Las mujeres con sobrepeso y obesidad no aumentan de peso en el primer trimestre del embarazo. La obesidad se asoció con un bajo aumento de peso en el segundo y tere $r$ trimestre del embaraø.

La correlación entre la ganancia de peso durante el embarazo y la concentración de hemoglobina se evaluó en mujeres embarazadas (Figura 2). Una mayor ganancia de peso durante el embarazo se correlacionó con una menor concentración de hemoglobina $\left(\mathrm{R}^{2}=\right.$ 0.963; $p<0.001)$.

\section{DISCUSIÓN}

La obesidad es considerada una afección proinflamatoria caracterizada por la presencia de inflamación sistémica crónica de bajo grado (Yanoff y col, 2008). Un número creciente de estudios han sugerido que la inflamación 
relacionada con la obesidad puede conducir a un defecto de la disponibilidad del hierro similar a la anemia por inflamación, donde la hepcidina se ha propuesto como mediador clave (Villarroel y col, 2013). Previamente, se ha demostrado que esta hormona se sobre expresa en la obesidad y se correlaciona con un bajo nivel de hierro en personas obesas (Yanoff y col, 2007), sin embargo no ha habido estudios que describan el efecto de la obesidad materna en el estado del hierro.

En este estudio decidimos estudiar la relación entre la obesidad pregestacional y el riesgo de tener una reducción de la hemoglobina en cada uno de los trimestres de mujeres embarazadas que residen a baja y gran altitud en el Perú durante el periodo 2012 al 2017. Los resultados mostraron lo contrario pues las gestantes con obesidad presentaban más bien un mayor nivel de hemoglobina en la gestación. Al evaluar, los niveles de hemoglobina corregida y no corregida según el índice de masa corporal (IMC), contrariamente a la hipótesis que planteamos, nuestros resultados demostraron que a medida que el IMC incrementaba también se incrementaba la concentración de hemoglobina corregida y no corregida $(p<0.0001)$.

Durante el embarazo de una mujer obesa se espera una mayor hemoconcentración porque la masa grasa no está bien perfundida en comparación con la masa magra corporal (Vricella y col, 2015).

Además, evaluamos si este patrón de aumento de hemoglobina en mujeres embarazadas obesas se mantuvo al evaluarlo por trimestre. Nuestros resultados demostraron lo que se describió anteriormente, las mujeres embarazadas con sobrepeso y obesidad tienden a aumentar los niveles de hemoglobina en comparación con las mujeres embarazadas de bajo y peso normal, independientemente del trimestre en que se encuentren.

Según los hallazgos de Ribot y col, las mujeres embarazadas obesas tenían menos probabilidades de tener anemia, pero si una mayor probabilidad de tener hemoconcentración. En consecuencia, nuestros hallazgos sugieren que las mujeres embarazadas con sobrepeso y obesidad tienden a aumentar los niveles de hemoglobina en comparación con las mujeres embarazadas de peso normal y bajo peso. Sin embargo, lejos de esto ser positivo para la gestante, es considerado un factor de riesgo ya que se ha demostrado que si se tiene un IMC elevado hay un alto riesgo de sufrir anemia postparto (Bodnar y col, 2004).

La mayoría de las poblaciones que viven a gran altitud muestran un aumento en la concentración de hemoglobina como resultado de aumento de la actividad eritropoyética como mecanismo para compensar un efecto de hipoxia tisular consecuencia de la baja presión barométrica a la cual están sometidos (Gonzales y col 2011; Gonzales y col, 2014). Sin embargo, también hay una reducción del volumen plasmático (Sanchez y col, 1970, Gonzales y col, 2017), algo muy parecido a lo que se ha encontrado que sucede en mujeres embarazadas obesas. Observamos en nuestros resultados que, a mayor altitud, ya sea teniendo en cuenta el trimestre o el IMC, las concentraciones de hemoglobina eran mayores en comparación con las de baja altitud. Esto demuestra que efectivamente la altura junto con la obesidad tiene un doble impacto en el aumento de la concentración de $\mathrm{Hb}$, lo cual podría llevar a la gestante a una serie de complicaciones y así mismo, repercutir sobre la salud del recién nacido. Este tópico de combinar obesidad y vida en las grandes alturas en la gestación ha sido poco estudiada y merece ser un tema donde se deban profundizar las futuras investigaciones.

Se sabe que las mujeres embarazadas obesas tienen una menor ganancia de peso (Vricella et al 2015), ya que estaría asociado con una disminución en el volumen sanguíneo lo cual llevaría a producir hemoconcentración. Evaluamos la ganancia de peso según el IMC y nuestros resultados confirmaron que las mujeres embarazadas con obesidad aumentaban solo la mitad del aumento de peso observado en mujeres embarazadas con IMC normal $(6.22 \mathrm{~kg}$ frente a $3.13 \mathrm{~kg}, \mathrm{p}$ $<0.0001)$. La hemoconcentración ha sido asociada a un aumento en el riesgo de preeclampsia y de nacimiento de pequeños para edad gestacional (Khoigani y col, 2012), y un riesgo elevado de mortalidad materna y perinatal.

La ganancia excesiva de peso gestacional también puede precipitar el desarrollo de la anemia por deficiencia de hierro materna (AIF) en la gestación posterior (yanoff et al 2007). Ante esta afirmación decidimos evaluar la correlación entre la ganancia de peso y la concentración $\mathrm{de} \mathrm{Hb}$ en gestantes, y nuestros resultados demostraron lo indicado, que una mayor ganancia de peso está asociado a una disminuc ón en las o ne ntrać ones de hemoglobina de la gestante.

En conclusión, un mayor IMC está asociado a un aumento de la concentración de hemoglobina en la gestante independientemente del trimestre en el que se encuentre. Por otro lado, la excesiva ganancia de peso demostró estar asociada a una menor concentración de hemoglobina repercutiendo principalmente en las gestantes de bajo y normal peso. Sin embargo, es necesario medir el volumen plasmático de las gestantes con la finalidad de evitar malinterpretaciones de la real concentración de hemoglobina en gestantes con obesidad y sobrepeso.

Financiamiento: Autofinanciado.

Conflicto de interés: Los autores declaran no tener algún conflicto de intereses. 


\section{REFERENCIAS BIBLIOGRÁFICAS}

1. Aigner E, Feldman A, Datz C. Obesity as an Emerging Risk Factor for Iron Deficiency. Nutrients 2014, 6, 3587-3600

2. Barrera $C$ and Germain A. Obesity and pregnancy. Rev. Med. 2012; 23(2) 154 -158.

3. Bodnar L, Slega-Riz A, Gogswell M. High prepregnancy $\mathrm{BMI}$ increases the risk of postpartum anemia.Obes Res. 2004;12:941-948

4. De Benoist B, McLean E, Egli I, Cogswell M. WHO; Geneva, Switzerland: 2008. Worldwide Prevalence of Anaemia 19932005: WHO Global Database on Anaemia.http://apps.who.int/ iris/bitstream/10665/43894/1/9789241596657_eng.pdf

5. Dimasuay KG, Boeuf $P$, Powell TL, Jansson T. Placental responses to changes in the maternal environment determine fetal growth. Front Physiol 2016,7: 1-9,

6. Gonzales GF, Fano D, Vásquez-Velásquez C. Necesidades de investigación para el diagnóstico de anemia en poblaciones de altura. Rev Peru Med Exp Salud Publica. 2017;34(4):699708.

7. Gonzales GF, Tapia V, Gasco M. Correcting haemoglobin cut-offs to define anaemia in high-altitude pregnant women in Peru reduces adverse perinatal outcomes. Arch Gynecol Obstet.2014;290(1):65-74.

8. Gonzales GF. Hemoglobin and testosterone: importance on high altitude acclimatization and adaptation. Rev Peru Med Exp Salud Publica 2011, 28:92-100

9. Kordas K, Fonseca Centeno ZY, Pachón H, Jimenez Soto AZ. Being overweight or obese is associated with lower prevalence of anemia among Colombian women of reproductive age. J. Nutr. 2013;143:175-181.

10. Khoigani MG, Goli S, Hasanzadeh A. The relationship of hemoglobin and hematocrit in the first and second half of pregnancy with pregnancy outcome. Iran J Nurs Midwifery Res. 2012 Feb;17(2 Suppl 1):S165-70.
11. Metz J, Levin N, Hart D. Effect of altitude on the body/ venous haematocrit ratio. Nature 1962, 194:483 23.

12. Ribot B, Ruiz-Diez F, Abajo S, March G, Fargas F, Arija V. Prevalence of anaemia, risk of haemoconcentration and risk factors during the three trimesters of pregnancy. Nutr Hosp 2018; 35:123-130.

13. Sanad M, Osman M, Gharib A. Obesity modulate serum hepcidin and treatment outcome of iron deficiency anemia in children: A case control study. Ital J Pediatr 2011; $37: 34$.

14. Sanchez C, Merino C, Figallo M. Simultaneous measurement of plasma volume and cell mass in polycythemia of high altitude. J Appl Physiol 1970.28:775-778.

15. Villarroel P,Arredondo M, Olivares M.Anemia de las enfermedades crónicas asociada a obesidad: papel de la hepcidina como mediador central. Rev Med Chile 2013; 141: 887-894

16. Vricella L, Louis J, Chien E, Mercer B. Blood volume determination in obese and normal weight gravidas: The Hydroxyethyl Starch Method. American journal of obstetrics and gynecology. 2015;213(3):

17. West C, Sasser J, Baylis C. The enigma of continual plasma volume expansion in pregnancy: critical role of the renin-angiotensinaldosterone system. Am J Physiol Renal Physiol. 2016.

18. WHO. Iron Deficiency Anemia. Assessment, Prevention and Control. In: A Guide for Progamme Managers. Geneva: World Health Organization; 2001.

19. Yanoff L, Menzie C, Denkinger B, Sebring N, McHugh T, Remaley A, et al. Inflammation and iron deficiency in the hypoferremia of obesity. Int J Obes (Lond) 2007;31(9):1412-1419.

\section{Correspondencia:}

Dirección: Paola Olavegoya

Email: diego.fano.s@upch.pe 